\title{
Emergent patterns of localized damage as a precursor to catastrophic failure in a random fuse network
}

\author{
S. Lennartz-Sassinek ${ }^{*}$ \\ School of Engineering and School of Geosciences, University of Edinburgh, Edinburgh, EH9 3JL, United Kingdom and \\ Institute for Geophysics and Meteorology, University of Cologne, Cologne, Germany \\ M. Zaiser \\ School of Engineering, University of Edinburgh, Edinburgh, EH9 3JL, United Kingdom and Insitute for Materials Simulation, \\ University of Erlangen-Nuremberg, Dr-Mack-Strasse 77, 90762 Fürth, Germany \\ I. G. Main \\ School of Geosciences, University of Edinburgh, Edinburgh, EH9 3JL, United Kingdom \\ C. Manzato \\ Department of Applied Physics, Aalto University, School of Science, PO Box 14100, FI-00076 Aalto, Finland \\ S. Zapperi \\ CNR-IENI, Via R. Cozzi 53, 20125 Milano, Italy and ISI Foundation, Via Alasso 11/C, 10126 Torino, Italy
}

(Received 16 January 2013; published 18 April 2013)

\begin{abstract}
We study the failure of disordered materials by numerical simulations of the random fuse model. We identify emergent patterns of localized damage prior to catastrophic failure by statistically averaging the density of damage around the eventual failure nucleation point. The resulting pattern depends on fracture density and obeys the same scaling relations as would be expected for the stress field generated by a critical crack nucleating in a finite, disorder-free effective medium of varying size. The growth of this critical crack absorbs preexisting clusters according to a well-defined scaling relation. Unfortunately, in single model runs such precursory signals are not obvious. Our results imply that reliable and accurate prediction of failure in time-independent, microscopically brittle random materials in a real case is inherently problematic, and degrades with system size.
\end{abstract}

DOI: 10.1103/PhysRevE.87.042811

PACS number(s): 46.50.+a, 64.60.av, 71.23.An, 81.40.Np

\section{INTRODUCTION}

Finding ways to forecast the failure of materials, engineering structures, and geosystems is an important open problem of science and technology. Very often we know with great confidence that catastrophic failure is bound to occur, but we do not know exactly when and where. A classical example are earthquakes where the motion of tectonic plates gives rise to a slow stress build-up which is ultimately released by catastrophic fault rupture. Seismic activity is being monitored with high spatial and temporal resolution, but it is still unclear how the accumulated data can be used for predicting large seismic events [1]. In engineering constructions such as buildings, water dams, or mines [2-4], acoustic monitoring is used as an empirical method which provides remotely sensed information on the accumulation of damage. However, again no method is yet at hand which could reliably and accurately predict the imminent collapse of structures based on such data. The reason that leads us to think that a catastrophe will eventually occur lies in the gradual build-up of stress and concomitant accumulation of damage before the event. The key question is then to understand when this slow subcritical process will give way to a rapid acceleration and localization of damage, leading to catastrophic failure. A well-explored

\footnotetext{
*lennartzsassinek@gmail.com
}

pathway to try to answer this question is to search for early signatures of imminent failure in the time series of the precursory activity [5]. Despite the large research effort devoted to this issue, the outcome is still unsatisfactory, and it is still not clear whether systematic failure precursors with deterministic predictive power exist or not.

In this paper, we study damage localization before failure in a statistical model for the fracture of disordered media. To this end, we perform extensive simulations of the random fuse model (RFM) [6,7], probably the simplest model where disorder, stress enhancements, and stress interactions can be incorporated. The model has been successfully used to study fracture size effects arising from a random microcrack distribution [8-11] and from large notches [12]. In both cases it is possible to relate the geometrical and statistical properties of the cracks existing before failure to the actual value of the fracture stress. Here we consider some related questions: (i) can we identify localized signatures of the critical crack before it spreads in a catastrophic manner and (ii) how do these signatures depend on external and internal system parameters such as the system size, the applied stress, or the degree of structural disorder?

\section{MODEL}

The RFM represents a material undergoing quasibrittle failure by the electrical analog of a network of fuses. We 


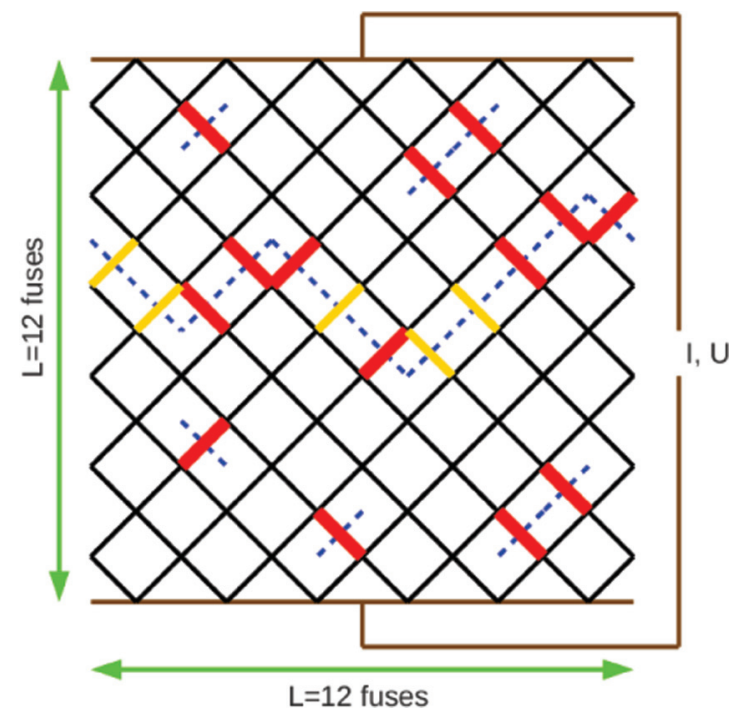

FIG. 1. (Color online) Sketch of a fuse network with applied global current $I$ and voltage $U$. Initially deleted bonds are indicated as thick red (dark) lines while the corresponding cracks, i.e., the barriers to the current, are indicated as dashed blue lines. In the simulation the applied current is increased, resulting in sequential breaking of the thick yellow (light) bonds until one crack (the critical crack) splits the network in two parts.

consider a square lattice of linear size $L$ in the $x y$ plane; see Fig. 1. The system is bounded in $y$ direction by top and bottom bus bars that impose a constant voltage, while periodic boundary conditions are imposed along the $x$ direction. The fuses all have unit resistance $R=1$ and fail at the critical local current $i_{c}=1$. This system is equivalent to a mechanical system undergoing antiplane shear deformation: The voltage represents the displacement in the $z$ direction, $V=u(x, y)$, while the current is related to the antiplane shear stress components by the relations $I_{x} / L=\sigma_{x z}$ and $I_{y} / L=$ $\sigma_{y z}$.

To introduce disorder, we delete randomly a fraction $f$ of the fuses. This introduces random spatial variations in the current passing each fuse, which are tantamount to a locally varying effective stress. At every simulation step, we evaluate all currents $i$ and irreversibly burn the fuse with the highest current if any $i \geqslant i_{c}$. Due to Kirchhoff's law, failure of a fuse results in a current redistribution which affects the whole lattice as the corresponding lattice Green's function scales like $G(x, y) \propto x / r^{2}[13]$.

Thus, the correlations are inherently long-range in contrast to those in cellular automata, where the local stress is only redistributed to nearest neighbors on failure. Because of current redistribution, burning of a fuse may result in a failure cascade as other fuses exceed their critical current. Damage accumulates as the global current $I$ increases through a sequence of such cascades until a connected fracture path disconnects the network in a global or catastrophic failure event. In the following we denote the fuse where the final failure cascade starts as the "critical bond" or "nucleation point," and the peak global current $I_{\text {peak }}$ at which this cascade initiates, divided by the bus bar length $L$, as the equivalent "critical stress" or "peak stress" $\sigma_{\mathrm{c}}=I_{\text {peak }} / L$.
We note that the present system exhibits a second mode of failure once the dilution fraction $f$ reaches the critical point $f=0.5$ for bond percolation. At the percolation critical point the system disconnects even without external stress, and, in the critical region close to this point, the damage patterns exhibit long-range correlations even in the initial state. In our investigation, which focuses on emergent damage patterns caused by the stress-driven correlated growth of damage clusters from short-range correlated disorder, we specifically exclude this regime and therefore consider only dilution fractions $f<0.3$, which are sufficiently far from the percolation limit.

\section{NUMERICAL SIMULATIONS}

\section{A. Damage pattern and critical crack phantom}

To identify localized precursors of failure, we consider the situation just before the peak global stress. We study the vicinity of the nucleation point and ask how local damage patterns there differ from those elsewhere in the system. Simple inspection of single realizations [see Fig. 2(a)] does not indicate any conspicuous features that would easily allow to identify the nucleation point before catastrophic failure. In particular, this point is not in general adjacent to the largest damage cluster, nor is it always located in the region of maximum damage density. Nevertheless, statistical analysis reveals a distinct pattern. We define an indicator function $g(\vec{r})$ which has the value $g=1$ at the location of a broken bond and $g=0$ at the location of an intact bond. A damage density function $\rho$ is then obtained by averaging $g$ over an ensemble of simulations (for our initial dilution disorder, this simply yields the constant damage density $\rho_{0}=f$ ). The key step is now to perform the average conditional on the location of the critical bond. Let $\vec{r}_{i}$ denote the site of the nucleation of the
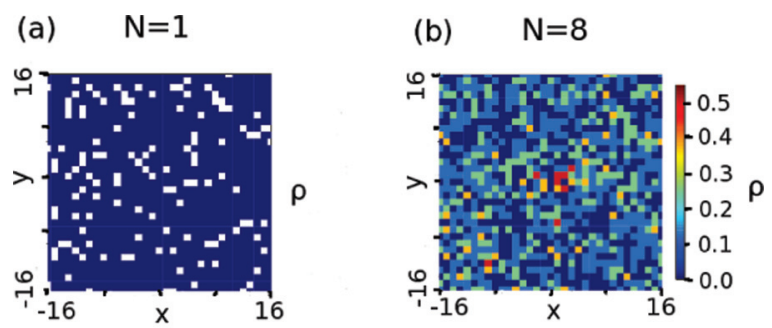

(c) $\quad \mathrm{N}=64$

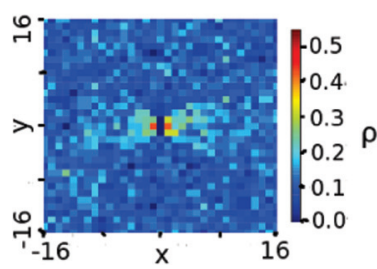

(d) $\quad \mathrm{N}>500$

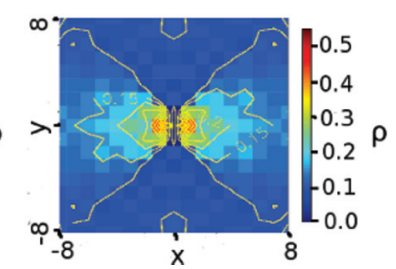

FIG. 2. (Color online) Damage density $\rho$ (in units of damage per fuse) around nucleation sites in systems of size $L=512$ with dilution $f=0.1$. (a) Single realization with binary color code (dark blue $=$ intact, white $=$ broken). (b-d) Damage density averaged over ensembles with increasing number $N$ of realizations, showing the emergence of a continuous damage distribution. The scale in (d) is reduced to better show details of the crack phantom pattern. 
critical system-breaking crack in simulation $i$. We define the conditional damage density as

$$
\rho(\vec{r})=\frac{1}{N} \sum_{i=1}^{N} g\left(\vec{r}-\vec{r}_{i}\right) .
$$

In performing the average, we have considered only simulations where the critical bond was located at distance larger than $L / 4$ from the upper or lower boundaries of the system. This ensures that the resulting damage pattern is not influenced by the presence of the top or bottom surface. Results are plotted in Fig. 2(b)-2(d). The conditional density pattern reveals an increased density of broken bonds to the left and right and a decreased damage density above and below the critical bond. This procedure of studying localized damage accumulation in terms of conditional averages is similar to the approach used in Ref. [12] where damage patterns around preexisting cracks were studied in order to establish statistical signatures of the fracture process zone surrounding the crack tips. This emergent damage pattern also occurs for the critical nucleation process. Similar to the process zones in Ref. [12], the damage accumulation zone surrounding the failure nucleation point becomes visible only after statistical averaging, while it cannot be identified in a single realization [compare Fig. 2(a) and 2(d)]. Nevertheless, as we will demonstrate, this zone exhibits properties similar to a critical crack, here we term it the "crack phantom."

To quantify properties of these crack phantoms we fit the excess damage density $\rho(\vec{r})-\rho_{0}$ by an empirical function

$$
\rho(\vec{r})-\rho_{0}=\phi(\theta) \exp \left[-\left(\frac{x^{2}+p y^{2}}{\xi^{2}}\right)^{\beta}\right] .
$$

The angle-dependent function $\phi$ with the symmetry properties $\phi(\theta)=\phi(-\theta)=\phi(\theta+\pi)$ accounts for the change in sign of the excess damage density as we move around the critical bond. The radial decay of the excess density is described by an anisotropic, stretched exponential function with characteristic range $\xi$. Approximating the angle-dependent function $\phi$ by a fourth-order polynomial in $\cos ^{2} \theta=x^{2} / r^{2}$ and performing fits over the range $0.05 \leqslant f \leqslant 0.3$ and for system sizes $32 \leqslant L \leqslant$ 512 , we find that the parameter $\beta \approx 0.33$ is approximately constant over the parameter range. The phantom size increases with increasing disorder as shown in Figs. 3(c), 3(d) and 4(a), and with increasing system size as shown in Figs. 3(a), 3(b) and 4(c). The latter dependency is well described by a logarithmic function, $\xi \propto \log (L)$ [dotted line in Fig. 4(c)]. In either case an increasing phantom size is accompanied by a decreasing failure stress [Figs. 4(b) and 4(d)].

\section{B. Relation to the failure stress}

To understand the role of the "phantom crack" in more quantitative terms we investigate how its characteristic size $\xi$ relates to the failure stress. A scaling relationship connecting strength with a characteristic length scale is standard in classical fracture mechanics where the length $a$ of a critical crack in an otherwise homogeneous system is connected to the failure stress $\sigma_{c}$ by $K_{\infty}=\sigma_{c} \sqrt{\pi a}$ where $K_{\infty}$ is the fracture toughness of the material [14]. If we replace $a$ with the phantom crack size $\xi$, this allows us to relate the logarithmic

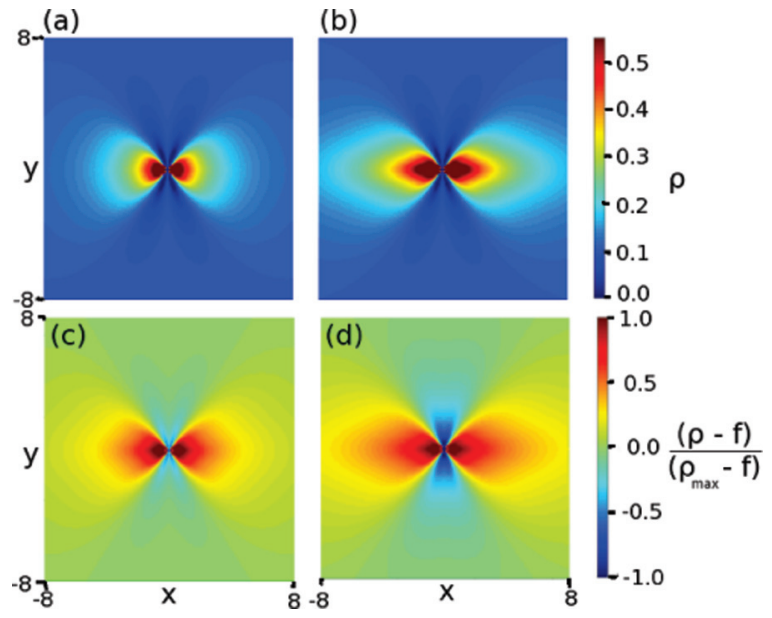

FIG. 3. (Color online) Upper row: Theoretical damage density $\rho$ (in units damage per fuse) calculated from Eq. (2) using the same scale as in Fig. 2 for $f=0.1, L=$ (a) 128, (b) 512. Panel (b) is a best fit to (d) in Fig. 2. Lower row: normalized density $(\rho-f) /\left(\rho_{\max }-f\right)$ for $L=256, f=$ (c) 0.15 , (d) 0.3 .

increase of $\xi$ with $L$ [Fig. 4(c)] to the decrease of the failure stress in proportion with $\sigma_{c} \propto 1 / \sqrt{\log (L)}$, which was first demonstrated by Kahng et al. [15] and recently confirmed over a much wider scale range by Manzato et al. [11]. In order to understand the dependence of $\sigma_{c}$ on the dilution fraction $f$, we need to account for the $f$ dependence of the fracture toughness, which for a brittle material can be expressed as $K_{\infty}=2 E \gamma$ where $E$ is the elastic modulus of the material and $\gamma$ is the specific surface energy. For a straight crack in our model, $\gamma=(1-f) / 4$ while the effective-medium result for the elastic modulus (the conductivity) is, except for a small vicinity of the percolation critical point, well described by the effective-medium result $E=(1-2 f)$ [16]. We arrive at

$$
\begin{aligned}
\sigma_{c}(f) & =K_{\infty}(f) / \sqrt{\pi \xi(f)}, \\
K_{\infty}(f) & \approx(1-f)(1-2 f) / 2,
\end{aligned}
$$

which gives the full line in Fig. 4(b). This theoretical line fits the data for correlation length very well. When we perform the same analysis for systems of different size, we find that strength is overestimated in the regime of small $L$. This can be corrected by taking into account the periodic boundary conditions in our simulations which lead to self-interactions across the lateral system boundaries, which enhance the stress concentration associated with the "crack phantoms." For a periodic array of mode III cracks with spacing $L$ and length $a$, the fracture toughness modifies to $K=K_{\infty} / \kappa(a / L)$ [17] where

$$
\kappa(a / L)=\sqrt{\frac{L}{\pi a} \tan \left(\frac{\pi a}{L}\right)} .
$$

Using this correction factor, we obtain the full line in Fig. 4(d), which is again in good agreement with the observed strength data. We conclude that the size of the crack phantom relates to the failure strength of the disordered system in a similar manner as the length of a critical crack to the failure strength of an otherwise homogeneous material: The "phantom" is the spectre of a nucleating critical crack. 

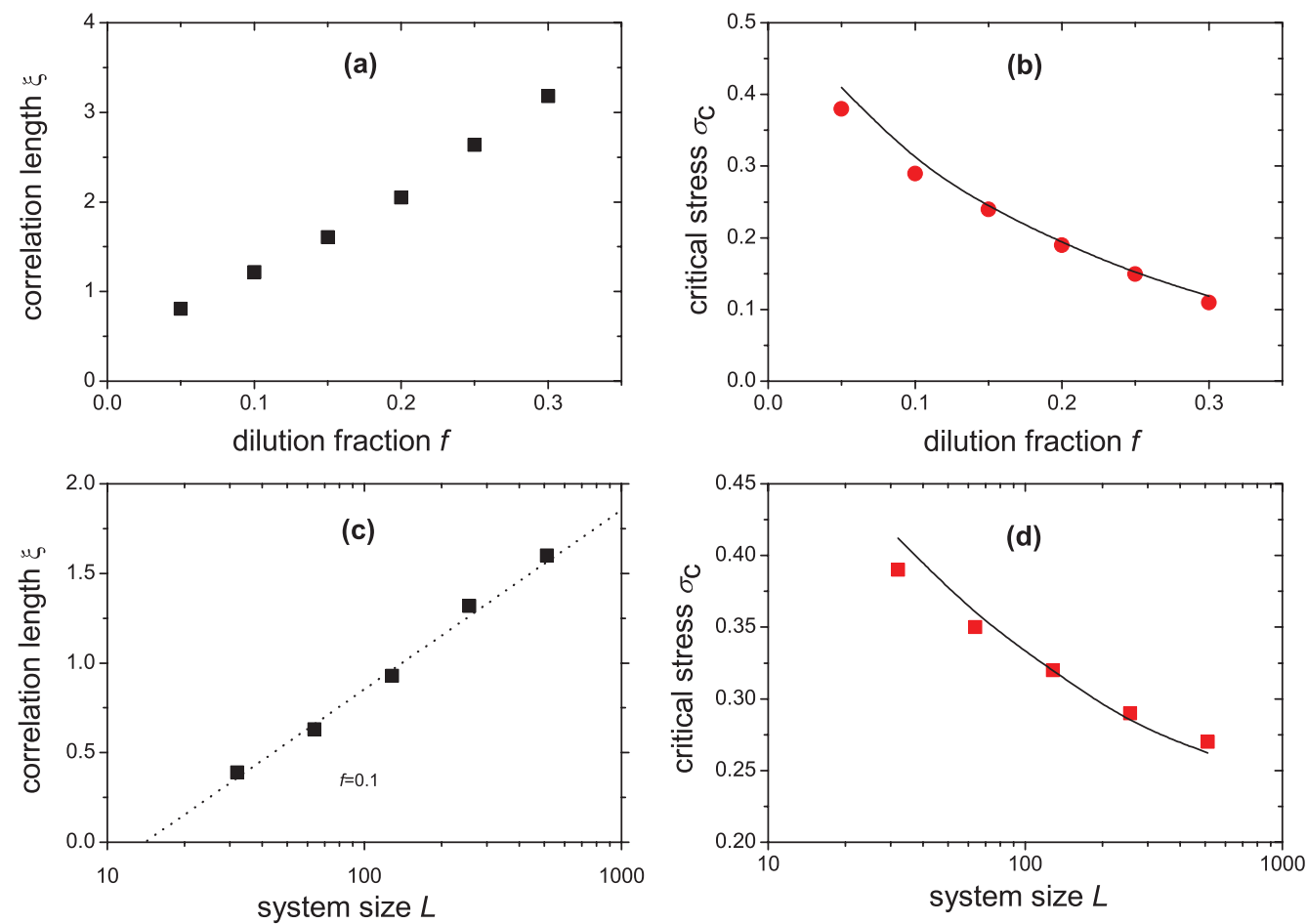

FIG. 4. (Color online) Dependence of phantom size $\xi(\mathrm{a}, \mathrm{c})$ (in units of fuses) and failure stress $\sigma_{c}(\mathrm{~b}, \mathrm{~d})$ (in units $i_{c} / L$ ) on dilution fraction $f(\mathrm{a}, \mathrm{b})$ (dimensionless) and system size $L$ (c, d) (in units of fuses); (a, b) system size $L=256$, dilution fraction $0.05 \leqslant f \leqslant 0.3$; (c, d) dilution fraction $f=0.1$, system size $32 \leqslant L \leqslant 512$. Dotted line in (c), $\xi \propto \log (L)$; full lines in (b) and (d), Predictions of failure stresses according to Eqs. (3) and (5), respectively, where $K_{\infty}$ is replaced by $K_{\infty} / \kappa(a / L)$ with $\kappa(a / L)$ according to Eq. (5).

\section{The formation of the final crack}

We now turn our attention to the question how the critical crack relates to the pattern of damage clusters prior to failure. Clusters are broken bonds (i.e., burned fuses) where the conjugate bonds (indicated as dashed lines in Fig. 1) are connected to each other. They can be envisaged as microcracks. We define the size $s$ of a cluster as the number of connected conjugate bonds and determine the numbers $N_{\text {before }}(s)$ and $N_{\text {after }}(s)$ directly before and after formation of the system-spanning crack. For large clusters, we find that $N_{\text {before }}(s) \propto \exp \left(-s / s_{1}\right)$

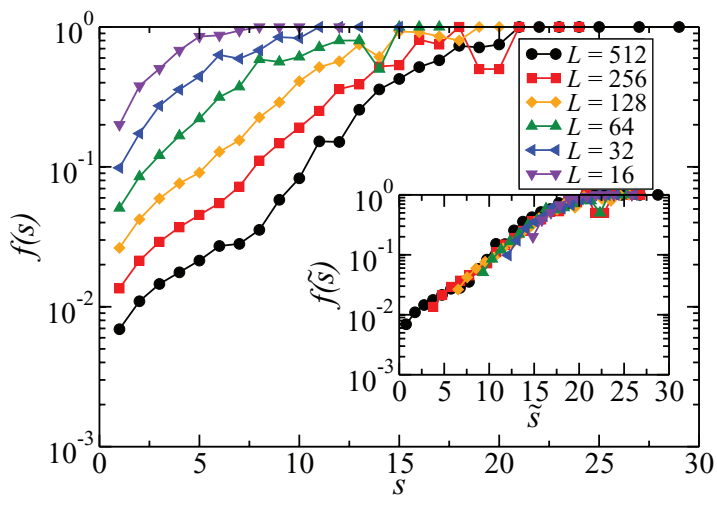

FIG. 5. (Color online) Relative fraction $f(s)$ of absorbed clusters of size $s$ directly before the peak current, which join the final systemwide crack. In the inset, the curves have been rescaled by Eq. (7) with $L_{0}=512$. The quantities $s, \tilde{s}, L$, and $L_{0}$ are given in units of fuses, whereas $f$ is a dimensionless quantity. where $s_{1} \approx 2$. The probability $f(s)$ that a preexisting cluster of size $s$ is absorbed into the spanning crack is then given by

$$
f(s)=\frac{N_{\text {before }}(s)-N_{\text {after }}(s)}{N_{\text {before }}(s)} .
$$

Figure 5 shows this probability and its system size dependence. For small and intermediate cluster sizes, the relative fraction of absorbed clusters increases exponentially, while it flattens to the asymptotic value $f(s)=1$ for very large clusters. To analyze the observed size dependence we make the following assumptions: (i) the spanning crack grows by connecting large clusters, the density of which is described by $N(s) / L^{2}=\exp \left(-s / s_{1}\right)$; (ii) the probability $f(s)$ depends only on the average number of clusters of size $s$ in the system: $f=f[N(s)]$. If these assumptions are fulfilled, the distributions can be collapsed by rescaling:

$$
\tilde{s}=s-2 s_{1} \ln \left(L / L_{0}\right),
$$

where $L_{0}$ is an arbitrary reference length (inset in Fig. 5 for $s_{1}=2$ ). Thus Fig. 5 demonstrate the importance of preexisting large clusters for the formation of the final system-spanning crack.

\section{CONCLUSIONS}

Our findings demonstrate the importance of localized damage clusters both for the location of the failure initiation point and for the subsequent propagation of the critical crack. Even though failure is not straightforwardly determined by the largest cluster (the critical bond is adjacent to the largest 
cluster in less than $50 \%$ of the simulations we analyzed), the properties of the large-cluster tail of the cluster size distribution are crucial for determining both the failure stress and the critical crack path. In the ensemble average, the nucleation sites are associated with a characteristic anisotropic damage pattern where damage is enhanced in the direction perpendicular and reduced in the direction parallel to the stress axis. Identifying this localized precursor pattern in the damage background requires averaging over different disorder realizations, using a posteriori knowledge of the location of the nucleation point. While the patterns are conspicuous in the ensemble average, they can therefore not be used for predicting failure in individual systems. In addition, the sizes of the localized precursor features we have been discussing (largest clusters, "crack phantom") scale only logarithmically with system size $L$, while in comparison the number of bonds and the overall number of damage clusters in the system scale with $L^{2}$. Thus, these features become less and less conspicuous in the large-system limit. Thus, the subcritical nature of failure associated with localized nuclei may render predictions based on such localized signatures an elusive goal in systems with a large ratio of system size to grain size. This may explain why precursors observed on a small scale in the laboratory are not observed systematically as earthquake precursers [18].

Our results have been obtained for two-dimensional random fuse models which represent an electrical analog of antiplane shear deformation. One may conjecture that our findings regarding the existence and scaling properties of "crack phantoms" carry over to three-dimensional systems, in a similar manner as Griffith-type scaling relations hold similarly for linear mode II/III cracks in two-dimensional systems and for penny-shaped cracks in three-dimensional systems. It will, however, remain a task for future investigations to verify this conjecture and to demonstrate general applicability of the "crack phantom" concept.

We have considered the case in which damage is irreversible and no healing occurs, while in real faults earthquakes can occur repeatedly in the same location. The random fuse model has been modified in the past to describe this behavior by inducing a permanent deformation after a fuse fails, instead of removing the fuse completely as in the case studied here [19-21]. In this cases damage mostly localizes into a shear band which corresponds to the minimum energy surface. After localization, power law-distributed avalanches of failure events occur along the shear band without apparent correlations. The observed phenomenology it is quite different from the one discussed here and therefore is not clear if the "crack phantom" idea can be applied to that case.

All of the results presented here are for an ideal case where all parameters are known. In real applications such as earthquake forecasting predictive power is also diminished by practical limitations in data sampling and resolution [1] and by systematically increased temporal nonlinearity in acceleration to system-sized failure as strain rate is decreased to natural rates due to chemically assisted weakening mechanisms [22].

\section{ACKNOWLEDGMENTS}

This work was supported by EPSRC and CNR as part of the ERA Complexity-NET pilot project LOCAT. We acknowledge for the data to the DEISA Consortium (EU projects FP6 RI-031513 and FP6 RI-222919) within the DEISA Extreme Computing initiative. We thank M. J. Alava, J. P. Sethna, and A. Shekhawat for useful discussions.
[1] I. G. Main, Nature (London) 385, 19 (1997).

[2] A. Petri, G. Paparo, A. Vespignani, A. Alippi, and M. Costantini, Phys. Rev. Lett. 73, 3423 (1994).

[3] A. Guarino, A. Garcimartin, and S. Ciliberto, Eur. Phys. J. B 6, 13 (1998).

[4] A. Guarino, S. Ciliberto, A. Garcimartin, M. Zei, and R. Scorretti, Eur. Phys. J. B 26, 141 (2002).

[5] J.-C. Anifrani, C. Le Floc'h, D. Sornette and B. Souillard, J. Phys. I (France) 5, 631 (1995).

[6] L. de Arcangelis, S. Redner, and H. J. Herrmann, J. Phys. Lett. (Paris) 46, 585 (1985).

[7] M. J. Alava, P. Nukala, and S. Zapperi, Adv. Phys. 55, 349 (2006).

[8] P. M. Duxbury, P. L. Leath, and P. D. Beale, Phys. Rev. B 36, 367 (1987).

[9] P. D. Beale and P. M. Duxbury, Phys. Rev. B 37, 2785 (1988).

[10] B. K. Chakrabarti and L. G. Benguigui, Statistical Physics of Fracture and Breakdown in Disordered Systems (Oxford University Press, New York, 1997).

[11] C. Manzato, A. Shekhawat, P. K. V. V. Nukala, M. J. Alava, J. P. Sethna, and S. Zapperi, Phys. Rev. Lett. 108, 065504 (2012).
[12] M. J. Alava, P. K. V. V. Nukala, and S. Zapperi, Phys. Rev. Lett. 100, 055502 (2008).

[13] A. Shekhawat, S. Papanikolaou, S. Zapperi, and J. P. Sethna, Phys. Rev. Lett. 107, 276401 (2011).

[14] B. Lawn, Fracture of Brittle Solids (Cambridge University Press, Cambridge, 1997).

[15] B. Kahng, G. G. Batrouni, S. Redner, L. de Arcangelis, and H. J. Herrmann, Phys. Rev. B 37, 7625 (1988).

[16] S. Kirkpatrick, Phys. Rev. Lett. 27, 1722 (1971).

[17] W. T. Koiter, Ing.-Arch. 28, 168 (1959).

[18] I. G. Main and M. Naylor, Eur. Phys. J. Special Topics 205, 183 (2012).

[19] P. A. Cowie, C. Vanneste, and D. Sornette, J. Geophys. Res. 98, 21809 (1993).

[20] P. Miltenberger, D. Sornette, and C. Vanneste, Phys. Rev. Lett. 71, 3604 (1993).

[21] C. B. Picallo, J. M. Lopez, S. Zapperi, and M. J. Alava, Phys. Rev. Lett. 105, 155502 (2010).

[22] I. O. Ojala, I. G. Main, and B. T. Ngwenya, Geophys. Res. Lett. 31, L24617 (2004). 